\title{
Optimal Treatment for the High-Risk Salivary Gland Cancer
}

\author{
Jeong-Soo Woo (1) \\ Department of Otorhinolaryngology-Head and Neck Surgery, Korea University College of Medicine, Seoul, Korea
}

Carcinomas of the major salivary glands constitute a heterogeneous group of rare malignant neoplasms, with 24 distinct histological types recognized by the World Health Organization classification. As a consequence of this heterogeneity, the literature lacks prospective studies with high-quality evidence from large groups of patients. This deficiency has made it challenging to achieve progress in the management of these patients. As a result, complete macroscopic surgical resection remains the definitive treatment of choice for patients with operable salivary gland cancer [1].

Multiple studies have identified poor prognostic factors of salivary gland cancer, such as high tumor grade, advanced $\mathrm{T}$ and $\mathrm{N}$ stages, advanced age, lymphovascular invasion, and positive surgical margins. Of these factors, tumor grade and nodal metastasis were found to be common independent factors in multivariate analyses with effects on survival, as well as locoregional spread and distant metastasis [2]. Regional metastatic disease has long been known to have prognostic and therapeutic implications [3]. Since high-grade tumors have a higher incidence of regional and distant metastasis, tumor grade has been described in the literature as one of the most compelling risk factors of treatment failure $[4,5]$.

Since several reports have shown a positive role of postoperative radiotherapy (RT) in patients with poor prognostic factors,

it is generally agreed that complete surgical resection of highrisk salivary gland cancer, followed by RT, is a rational approach to improve treatment outcomes [6]. The current National Comprehensive Cancer Network (NCCN) guidelines support surgery as the standard definitive treatment for these malignant neoplasms, with RT indicated for high-risk patients. Despite the addition of RT, the incidence of distant metastases in malignant salivary gland tumors is estimated to be around $20 \%-30 \%$ [7], and distant metastasis is the most common cause of treatment failure [8].

Although surgery followed by RT may result in excellent lo- coregional control, the high mortality rate associated with highrisk salivary gland cancer underscores the need for more aggressive treatment approaches, such as adjuvant chemotherapy or targeted therapy. Effective systemic therapies that address the high rate of distant metastasis must be developed to improve clinical outcomes. The rationale for adjuvant concurrent chemoradiotherapy (CCRT) for managing high-risk salivary gland malignancies draws from squamous cell carcinoma treatment.

However, few studies have aimed to assess the benefit of CCRT for high-risk salivary gland cancer treatment [9]. Since no randomized prospective study of CCRT has been conducted, this treatment option is still a subject of debate [10]. The current NCCN guidelines list CCRT as a category $2 \mathrm{~B}$ recommendation that can be considered for high-risk patients. Fortunately, the role of CCRT in the setting of high-risk salivary gland cancer is currently under investigation (RTOG 1008: a randomized phase II study of adjuvant concurrent radiation and chemotherapy versus radiation alone in resected high-risk malignant salivary gland tumors, clinical trial registry: NCT01272037). This is the first prospective trial attempting to answer this question for high-risk salivary gland cancer.

Combining future molecular-driven therapies with more sophisticated RT techniques and technologies may be another way to improve the outcomes of high-risk salivary gland tumors. Furthermore, given the likelihood that toxic effects related to treatment may contribute to negative outcomes, it is critical to identify which high-risk patients may benefit the most from a combined treatment modality.

\section{CONFLICT OF INTEREST}

No potential conflict of interest relevant to this article was reported. 


\section{ORCID}

Jeong-SooWoo https://orcid.org/0000-0001-8075-0976

\section{REFERENCES}

1. Schroeder U, Groppe D, Mueller RP, Guntinas-Lichius O. Parotid cancer: impact of changes from the 1997 to the 2002 American Joint Committee on Cancer classification on outcome prediction. Cancer. 2008 Aug;113(4):758-64.

2. Mifsud MJ,Tanvetyanon T, Mccaffrey JC, Otto KJ, PadhyaTA, Kish J, et al. Adjuvant radiotherapy versus concurrent chemoradiotherapy for the management of high-risk salivary gland carcinomas. Head Neck. 2016 Nov;38(11):1628-33.

3. Xiao CC, Zhan KY,White-Gilbertson SJ, DayTA. Predictors of nodal metastasis in parotid malignancies: a national cancer data base study of 22,653 patients. Otolaryngol Head Neck Surg. 2016 Jan; 154(1):121-30.

4. Haderlein M, Scherl C, Semrau S, Lettmaier S, UterW, Neukam FW, et al. High-grade histology as predictor of early distant metastases and decreased disease-free survival in salivary gland cancer irrespective of tumor subtype. Head Neck. 2016 Apr;38 Suppl 1:E2041-8.

5. Chen MM, Roman SA, Sosa JA, Judson BL. Histologic grade as prognostic indicator for mucoepidermoid carcinoma: a population- level analysis of 2400 patients. Head Neck. 2014 Feb;36(2):158-63.

6. Jeannon JP, Calman F, Gleeson M, McGurk M, Morgan P, O'Connell $\mathrm{M}$, et al. Management of advanced parotid cancer: a systematic review. Eur J Surg Oncol. 2009 Sep;35(9):908-15.

7. Franchi A, Gallo O. Distant metastases and management. In: Bradley PJ, Guntinas-Lichius O, editors. Salivary gland disorders and diseases: diagnosis and management. Stuttgart:Thieme; 2011. p. 341-9.

8. Kim YH, Chung WK, Jeong JU, Cho IJ, Yoon MS, Song JY, et al. Evaluation of prognostic factors for the parotid cancer treated with surgery and postoperative radiotherapy. Clin Exp Otorhinolaryngol. 2020 Feb;13(1):69-76.

9. Jegadeesh N, Liu Y, Prabhu RS, Magliocca KR, Marcus DM, Higgins $\mathrm{KA}$, et al. Outcomes and prognostic factors in modern era management of major salivary gland cancer. Oral Oncol. 2015 Aug;51(8): 770-7.

10. Amini A, Waxweiler TV, Brower JV, Jones BL, McDermott JD, Raben $\mathrm{D}$, et al. Association of adjuvant chemoradiotherapy vs radiotherapy alone with survival in patients with resected major salivary gland carcinoma: data from the national cancer data base. JAMA Otolaryngol Head Neck Surg. 2016 Nov;142(11):1100-10. 